\title{
Saisonale Schwankungen des Serum-IgE-Spiegels und deren mögliche Bedeutung für die Berechnung der therapeutischen Dosis von Omalizumab (rhuMab-E25, anti-IgE)
}

K. M. Beeh' ${ }^{1}$
J. Beier ${ }^{2}$
R. Buhl' ${ }^{1}$

\author{
Seasonal Variations of Serum-IgE and Potential Impact on Dose-Calculation \\ of Omalizumab (rhuMab-E25, anti-IgE)
}

\section{Zusammenfassung}

Hintergrund: Mit dem monoklonalen anti-IgE-Antikörper Omalizumab steht eine Erweiterung der Behandlung des allergischen Asthma bronchiale zur Verfügung. Die Dosierung von Omalizumab erfolgt nach Serum-IgE und Körpergewicht. Eine nahezu vollständige Suppression des Serum-IgE ist für den Therapieerfolg wichtig. Viele Asthmatiker weisen neben perennialen auch saisonale Sensibilisierungen auf, und der saisonale IgE-„Boost“ könnte eine Dosisanpassung erforderlich machen, bzw. den Therapieerfolg negativ beeinflussen. Methode: Wir untersuchten Gesamt-IgE-Spiegel und spezifisches (Lieschgras g6) IgE bei 17 Patienten mit saisonaler allergischer Rhinitis und/oder saisonalem Asthma vor und während der Gräserpollensaison. Anhand des Dosierungsschemas für Omalizumab errechneten wir hypothetische Dosen vor und während der Pollensaison. Ergebnisse: Das Gesamt-IgE der Patienten lag präsaisonal bei 89 (50-178) $\mathrm{kU} / 1$ (geom. Mittelwert mit 95\% Konfidenzintervall) und stieg saisonal signifikant auf $126(63-251) \mathrm{kU} / \mathrm{l}$ an $(\mathrm{p}=0,0006)$. Das spezifische IgE stieg parallel hierzu von $11(6,3-19) \mathrm{kU} / \mathrm{l}$ auf $15,1(8,3-29) \mathrm{kU} / \mathrm{l}(\mathrm{p}=0,0013)$. Die errechneten präsaisonalen Dosierungen von Omalizumab waren: keine Dosierung (IgE $<30 \mathrm{kU} / \mathrm{l}): \mathrm{n}=2 ; 150 \mathrm{mg}$ alle 4 Wochen: $\mathrm{n}=7 ; 300 \mathrm{mg}$ alle 4 Wochen: $\mathrm{n}=2 ; 225 \mathrm{mg}$ alle 2 Wochen: $\mathrm{n}=4 ; 300 \mathrm{mg}$ alle 2 Wochen: $\mathrm{n}=1 ; 375 \mathrm{mg}$ alle 2 Wochen: $\mathrm{n}=1$. Auf der Grundlage der saisonalen IgE-Spiegel hätte sich für 5/17 Patienten eine Änderung der Omalizumab-Dosis in die nächsthöhere Dosisstufe ergeben, davon wären zwei Patienten aus dem Dosierungsbereich herausgefallen. Schlussfolgerung: Ein saisonaler Anstieg des Serum-IgE lässt sich bei Patienten mit Pollenallergie beobachten. Dieser wäre für die Mehrzahl der Patienten ohne Einfluss auf die Dosis-

\section{Abstract}

Background: The anti-IgE antibody Omalizumab has been approved for the treatment of perennial allergic asthma. Dosing of omalizumab is adjusted according to total IgE levels and body weight, and a near complete suppression of free IgE is deemed to be necessary for optimal efficacy. Many asthmatics, however, have additional sensitisations against seasonal allergens, e.g. pollen, and seasonal exposure may increase total and specific IgE levels ("boost"), thus leading to an imbalance between IgE levels and omalizumab with possible impact on therapeutic outcome. Methods: We studied serum total and specific (timothy grass) IgE levels in 17 patients with seasonal allergic rhinitis and/ or asthma prior to and during the grass pollen season. Based on total IgE levels, we then calculated hypothetical doses of omalizumab required for treatment at each time point. Results: During the pollen season, total IgE increased significantly from a preseasonal mean of $89(50-178) \mathrm{kU} / \mathrm{l}$ (geom. mean with $95 \%$ confidence interval) to $126(63-251) \mathrm{kU} / \mathrm{l}(\mathrm{p}=0.0006)$. Accordingly, specific IgE increased from $11(6.3-19) \mathrm{kU} / \mathrm{l}$ to $15.1(8.3-29) \mathrm{kU} / \mathrm{l}$ $(p=0.0013)$. Calculated doses of omalizumab based on pre-seasonal IgE levels were: no dosing $(\operatorname{IgE}<30 \mathrm{kU} / \mathrm{l}): \mathrm{n}=2 ; 150 \mathrm{mg}$ 4-weekly: $\mathrm{n}=7 ; 300 \mathrm{mg}$ 4-weekly: $\mathrm{n}=2 ; 225 \mathrm{mg}$ 2-weekly: $\mathrm{n}=4 ; 300 \mathrm{mg}$ 2-weekly: $\mathrm{n}=1 ; 375 \mathrm{mg}$ 2-weekly: $\mathrm{n}=1$. Based on seasonal IgE levels, doses of omalizumab would have changed in $5 / 17$ patients requiring the next possible dosing step. Of these, two patients would have fallen out of the current dosing scheme. Conclusions: A seasonal increase of serum total and specific IgE can be observed in patients with pollen allergy, although this increase would have no impact on omalizumab doses in the majority of patient. Individual variations, however, can be large and

Institutsangaben

${ }^{1}$ Schwerpunkt Pneumologie III. Medizinische Klinik, Universitätsklinikum Mainz

${ }^{2}$ INSAF Institut für Atemwegsforschung, Wiesbaden

Korrespondenzadresse

Dr. med. Kai-Michael Beeh · Schwerpunkt Pneumologie · III. Medizinische Klinik • Langenbeckstr. 1 .

55131 Mainz·E-mail: k.beeh@3-med.klinik.uni-mainz.de

Eingang: 11. Februar 2004 - Nach Revision akzeptiert: 12. April 2004

Bibliografie

Pneumologie 2004; 58: 546-551 @ Georg Thieme Verlag KG Stuttgart · New York

DOI $10.1055 / \mathrm{s}-2004-818483$

ISSN 0934-8387 
berechnung von Omalizumab, könnte im Einzelfall jedoch erhebliche Ausmaße haben und eine Dosiskorrektur erfordern. Ein therapeutisches Monitoring freier IgE-Spiegel unter anti-IgETherapie erscheint daher notwendig und sinnvoll. necessitate a dose correction. Therefore, therapeutic monitoring of free IgE levels during anti-IgE treatment appears as desirable tool.

\section{Hintergrund}

Das wachsende Verständnis pathogenetischer Prozesse im Rahmen der allergischen Entzündung hat zur Entwicklung neuer Therapieverfahren geführt. Bei allergischen Individuen liegt eine vermehrte Bereitschaft zur IgE-Synthese nach Kontakt mit ubiquitären, apathogenen Fremdproteinen wie Pollen, Hausstaubmilben etc. vor. Bei Allergenexposition und -bindung an IgE-Moleküle auf Effektorzellen (Mastzellen oder Basophile) in respiratorischen Epithelien (Nasen-, Bronchialschleimhaut) löst die Kreuzvernetzung („,bridging“) von IgE die Freisetzung proinflammatorischer Mediatoren und in der Folge klinische Symptome im Sinne einer Rhinitis bzw. eines Asthma bronchiale aus. Mit dem rekombinanten, humanisierten monoklonalen anti-IgEAntikörper rhuMab-E25 (Omalizumab, Xolair ${ }^{\circledR}$ ) existiert eine neuartige Therapieoption, die es erstmalig erlaubt, IgE als Schlüsselmolekül der allergischen Reaktionskaskade zu blockieren. Omalizumab bindet an das Fc-Fragment zirkulierender IgEAntikörper und verhindert die IgE-Bindung an hoch- und niedrig-affine IgE-Rezeptoren auf Mastzellen und Basophilen [1].

Omalizumab ist bislang in den USA und Australien zur Behandlung des mittelschweren oder schwergradigen allergischen Asthma bronchiale zugelassen. Der klinische Nutzen von Omalizumab in dieser Indikation [2 -4], aber auch bei Patienten mit allergischer Rhinitis [5-7] ist durch zahlreiche kontrollierte Studien belegt. Omalizumab senkt nach subkutaner Applikation dosisabhängig zirkulierende Serum-IgE-Spiegel durch Bildung von Omalizumab-IgE-Immunkomplexen. Für den klinischen Erfolg der anti-IgE-Therapie ist jedoch die adäquate Dosierung von entscheidender Bedeutung [8]. Frühe in vitro-Studien zeigen, dass erst eine Reduktion von zirkulierendem IgE um mehr als 99\% eine allergenvermittelte Kreuzvernetzung von IgE-Rezeptoren auf Effektorzellen wirksam unterbindet [9]. Daher berechnet sich die Dosierung von Omalizumab nach der mittels Serum-Gesamt-IgE und Körpergewicht abgeschätzten „IgE-Belastung“ des Organismus, um eine maximale Reduktion von freiem IgE zu erreichen [10].

Eine bislang noch offene Frage ist die Stabilität von Serum-IgE im Kurz- oder Langezeitverlauf, da größere Schwankungen der Zielgröße IgE eine Anpassung der Omalizumab-Dosis erforderlich machen, bzw. u.U. auch eine Dosisreduktion ermöglichen könnten. Dies ist angesichts der hohen Kosten des Antikörpers auch von ökonomischer Relevanz. Es konnte wiederholt gezeigt werden, dass insbesondere bei saisonalen Sensibilisierungen sowohl Gesamt- als auch spezifische IgE-Spiegel bei saisonaler Exposition („Boost“) deutlich ansteigen können [11,12]. Das optimale Instrument zur Steuerung der anti-IgE-Therapie stellt daher die Bestimmung des freien, d.h. nicht mit Omalizumab komplexierten IgE, dar. Freies IgE kann jedoch mit herkömmlichen IgE-Assays nicht nachgewiesen werden, da die Antikörper dieser Tests Epitope sowohl von freiem als auch komplexiertem IgE erkennen.

In der vorgelegten Untersuchung gingen wir der Frage nach, in welchem Maße Serumspiegel des Gesamt- und spezifischen IgE bei Patienten mit saisonaler Allergie schwanken, und inwiefern diese Schwankungen Auswirkungen auf eine hypothetische Therapie mit Omalizumab im Sinne einer Dosisanpassung haben könnten. Wir weisen nach, dass unter Verwendung des gängigen Dosierungsschemas saisonale Schwankungen des IgE-Spiegels für die überwiegende Mehrzahl der Patienten unerheblich wären, in Einzelfällen aber größere Schwankungen eine Dosisanpassung erforderlich machen würden.

\section{Methoden}

\section{Patienten}

Wir untersuchten 17 Patienten (mittleres Alter $37 \pm 10$ Jahre, 10 Männer) mit einer anamnestisch und klinisch gesicherten saisonalen allergischen Rhinitis aufgrund einer Sensibilisierung gegen Gräserpollen. Alle Patienten hatten positive Prick-Hautteste (Quaddelgröße $>5 \mathrm{~mm}$ ) gegen Lieschgras bzw. Gräsermischung und erhöhtes spezifisches IgE (CAP/FEIA-RAST-Klasse $>=2$ ) gegen Lieschgras. 4 der 17 Patienten hatten zudem typische Begleitsymptome eines leichtgradigen, saisonalen Asthma bronchiale. Keiner der Patienten erhielt während der Pollensaison systemische oder topische Glukokortikoide. Zur Behandlung allergischer Symptome verwendeten alle Patienten kurz- oder mittellangwirkende Antihistaminika oder nasale Vasokonstriktoren bei Bedarf, sowie bei asthmatischen Beschwerden kurzwirksame $\beta$-Sympathomimetika. Die Blutentnahmen zur Bestimmung der IgE-Spiegel erfolgten im Rahmen ambulanter Untersuchungen in der Ambulanz der Universitätsklinik Mainz. Alle Patienten wurden über den Zweck der zweiten, saisonalen Blutentnahme aufgeklärt und gaben ihr schriftliches Einverständnis. Ein Ethikvotum war nach Rücksprache mit der lokalen Ethikkommission für diese Verlaufsbeobachtung nicht erforderlich.

\section{Bestimmung von Gesamt- und spezifischem IgE}

Die Bestimmung des Gesamt- und spezifischen IgE erfolgte mittels enzymatischen Nachweis unter Verwendung des Pharmacia CAP/FEIA Immunoassays. Zur Bestimmung des spezifischen IgE diente Lieschgras (g6) als Allergen. Die präsaisonale Blutentnahme erfolgte bei allen Patienten mindestens 6 Wochen vor dem erwarteten Beginn der Gräserpollensaison (April/Mai). Die zweite, saisonale Blutentnahme erfolgte schließlich mindestens 4 Wochen nach Beginn der Gräserpollensaison, jeweils während hoher Pollenbelastung. Der saisonale Pollenflug wurde über öffentliche Polleninformationsdienste verfolgt. 
Berechnung der hypothetischen Dosierung von Omalizumab Die Berechung der hypothetischen Dosis von Omalizumab jeweils vor und während der Pollensaison erfolgte unter Verwendung des Dosierungsschemas des Beipackzettels für Xolair ${ }^{\circledR}$ aus Australien [10] und ist in Tab.1 angegeben. Zur Berechung werden Gesamt-IgE und Körpergewicht (kg) des Patienten benötigt. Bei Patienten, deren IgE-Wert oder Körpergewicht präsaisonal oder saisonal außerhalb des Schemas lagen, wurde die Formel: „Körpergewicht $(\mathrm{kg}) \times$ Gesamt-IgE $(\mathrm{kU} / \mathrm{l}) \times 0,008 \mathrm{mg} / \mathrm{kg} /(\mathrm{kU} /$ $\mathrm{l}$ ) = Dosis (mg) alle 2 Wochen“ angewendet [10].

\section{Statistische Methoden}

Alle Analysen wurden mit dem Programm STATA Version 5.0 (Stata Corp., College Station, TX, USA) für Personal Computer durchgeführt. Sofern nicht anders erwähnt, erfolgt die Angabe der Daten als arithmetische Mittelwerte mit Standardabweichung (SD) oder geometrische Mittelwerte mit 95\% Konfidenzintervall [13]. Mittels Kolmogorov-Smirnov-Test wurde eine Normalverteilung der Parameter ermittelt bzw. ausgeschlossen. Präsaisonale und saisonale Parameter wurden mittels gepaartem Student-t-Test für normal verteilte und Mann-Whitney-U-Test für nicht-normal verteilte Werte beurteilt. Korrelationen wurden mittels nicht-parametrischem Rang-Korrelationskoeffizienten nach Spearman (rho) ermittelt. Irrtumswahrscheinlichkeiten mit einem $\mathrm{p}$-Wert von $<0,05$ wurden generell als statistisch signifikant angesehen.

\section{Ergebnisse}

Das präsaisonale Gesamt-IgE aller Patienten lag bei 89 (50-178) $\mathrm{kU} / \mathrm{l}$ (geometrischer Mittelwert mit 95\% Konfidenzintervall), das spezifische IgE bei $11(6,3-19) \mathrm{kU} / \mathrm{l}$. Das spezifische IgE repräsentierte 12,25 (7,4-20)\% des Gesamt-IgE. Es bestand eine signifikante Korrelation zwischen der Höhe des Gesamt-IgE und dem spezifischen $\operatorname{IgE}(\mathrm{rho}=0,73, \mathrm{p}=0,001)$. Auf der Grundlage von IgE und Körpergewicht ergab sich für die 17 Patienten präsaisonal folgendes Dosierungsmuster für Omalizumab:
- keine Dosis (IgE unterhalb des Mindestwertes von $30 \mathrm{kU} / \mathrm{l}$ ): $\mathrm{n}=2$

- $150 \mathrm{mg}$ Omalizumab alle 4 Wochen s.c.: $\mathrm{n}=7$

- $300 \mathrm{mg}$ Omalizumab alle 4 Wochen s.c.: $\mathrm{n}=2$

- $225 \mathrm{mg}$ Omalizumab alle 2 Wochen s.c.: $\mathrm{n}=4$

- $300 \mathrm{mg}$ Omalizumab alle 2 Wochen s.c.: $\mathrm{n}=1$

- $375 \mathrm{mg}$ Omalizumab alle 2 Wochen s.c.: $\mathrm{n}=1$

- keine Dosis (IgE oberhalb des Höchstwertes von $1300 \mathrm{kU} / \mathrm{l}$ ): $\mathrm{n}=0$

Während der Pollensaison stieg das Serum-IgE bei 16/17 Patienten an. Der saisonale Mittelwert war mit 126,1 (63-251) kU/l signifikant höher als präsaisonal $(\mathrm{p}=0,0006)$. Parallel hierzu stieg das spezifische IgE im Mittel auf 15,1 (8,3-28) kU/l, an ( $p=0,0013$ ), ein Anstieg war bei 14/17 Patienten nachweisbar (Abb.1a und 1b). Der prozentuale Anteil des spezifischen IgE am Gesamt-IgE blieb während der Saison unverändert bei 12 $(7,2-20) \%(p=0,78)$. Der relative Anstieg des Gesamt-IgE war hochsignifikant mit dem relativen Anstieg des spezifischen $\operatorname{IgE}$ korreliert ( $r h o=0,75, p=0,001$ ) (Abb. 2). Auf der Grundlage der saisonalen IgE-Spiegel bei konstantem Körpergewicht hätte bei 5/17 (29\%) Patienten eine Änderung der Omalizumab-Dosis resultiert (Abb. 3). Von diesen fielen zwei Patienten saisonal aus dem Dosisschema heraus. Auch nach Berechnung über die Alternativformel (s. Methoden) lagen beide Patienten über dem empfohlenen Dosierungsschema (max. $375 \mathrm{mg}$ alle 2 Wochen). Hinsichtlich der individuellen Variabilität der IgE-Spiegel konnten wir bei unseren Patienten keinen Unterschied zwischen alleinigen Rhinitikern und Patienten mit Rhinitis und Asthma beobachten.

\section{Diskussion}

Mit dem anti-IgE-Antikörper Omalizumab steht seit kurzem eine Erweiterung der therapeutischen Optionen bei allergischen Erkrankungen zur Verfügung. Omalizumab ist in den USA und Australien zur Behandlung des mittelschweren bis schweren al-

Tab. 1 Dosierungsschema für Omalizumab nach Körpergewicht und Gesamt-IgE aus dem Beipackzettel (Australien)

\begin{tabular}{|c|c|c|c|c|c|c|c|}
\hline \multicolumn{8}{|c|}{ Körpergewicht (kg) } \\
\hline Gesamt-IgE & $>30-40$ & $>40-50$ & $>50-60$ & $>60-70$ & $>70-80$ & $>80-90$ & $>90-150$ \\
\hline$>100-200$ & 150 & 300 & 300 & 300 & 300 & 300 & 450 \\
\hline$>200-300$ & 300 & 300 & 300 & 450 & 450 & 450 & 600 \\
\hline$>300-400$ & 300 & 450 & 450 & 450 & 600 & 600 & \\
\hline$>600-700$ & 450 & 600 & 750 & & & & \\
\hline$>700-800$ & 600 & 750 & & & & & \\
\hline$>800-900$ & 600 & 750 & & & & & \\
\hline$>900-1000$ & 750 & & & & & & \\
\hline$>1000-1100$ & 750 & & & & & & \\
\hline
\end{tabular}

Dosen $\leq 300 \mathrm{mg}$ alle 4 Wochen werden $1 \times$ alle 4 Wochen appliziert

Dosen > 300 mg werden auf zwei equivalente Dosen aufgeteilt und alle 2 Wochen appliziert 

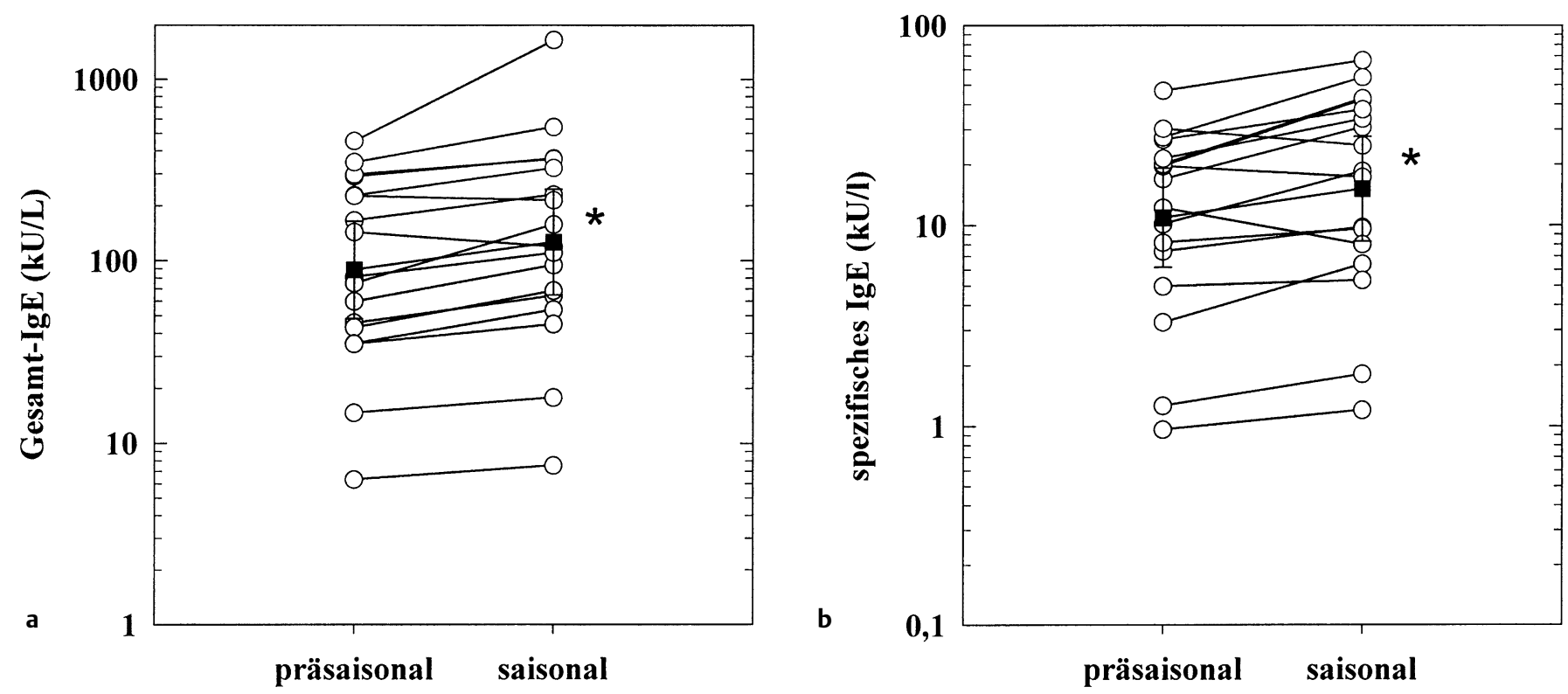

Abb. 1a und b Serumkonzentrationen von (a) Gesamt- und (b) spezifischem (Lieschgras) IgE bei Patienten mit Gräserpollenallergie vor und während der Pollensaison. ${ }^{*} \mathrm{p}=0,0006$, und ${ }^{*} \mathrm{p}=0,0013$ gegenüber dem präsaisonalen Wert.

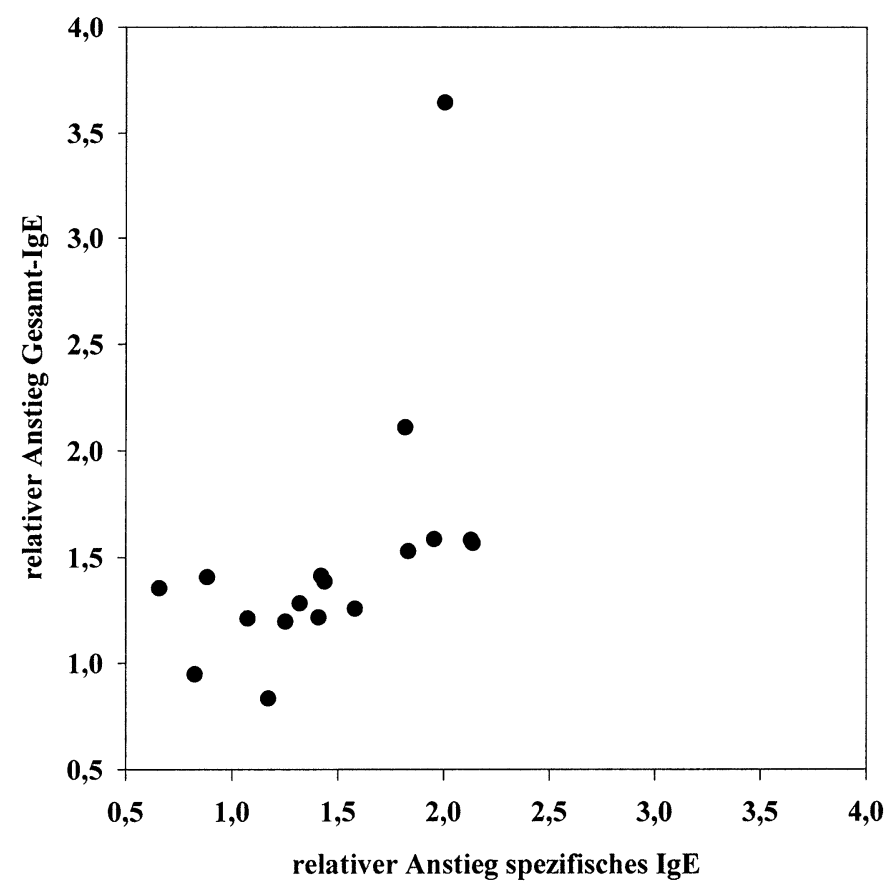

Abb. 2 Korrelation zwischen relativem, saisonalen Anstieg von Gesamt- und spezifischem IgE. rho $=0,75, p=0,001$.

lergischen Asthma bronchiale zugelassen. Die Dosierung von Omalizumab richtet sich nach der Höhe des Gesamt-IgE-Spiegels und dem Körpergewicht des Patienten. Dementsprechend sind in der Langzeittherapie notwendige Anpassungen der Omalizumab-Dosis aufgrund von Schwankungen des Körpergewichtes und IgE-Spiegeln theoretisch denkbar.

Für den therapeutischen Erfolg der Omalizumab-Behandlung ist eine weitestgehend vollständige Suppression des freien IgE unabdingbar. Frühe Studien bei Patienten mit allergischer Rhinitis haben eine klare Abhängigkeit der Symptomverbesserung vom Ausmaß der IgE-Reduktion zeigen können [8]. Es ist bislang noch nicht vollständig beantwortet, inwiefern temporäre individuelle Schwankungen des IgE-Spiegels den Therapieeeffekt von Omalizumab beeinflussen bzw. eine Dosisanpassung notwendig machen könnten. In der vorgelegten Arbeit untersuchten wir Gesamt-IgE-Spiegel und spezifisches IgE bei Patienten mit saisonaler allergischer Rhinitis vor und während der Gräserpollensaison. Für unsere Untersuchungen wählten wir bewusst Patienten mit saisonaler allergischer Rhinitis. Wenngleich diese Patienten primär nicht in die Indikation von Omalizumab fallen, so weisen doch viele Asthmatiker zusätzlich zu perennialen auch saisonale Sensibilisierungen und Ko-Morbidität mit allergischer Rhinitis auf [14]. Die von uns untersuchten Patienten hatten zudem den Vorteil einer fehlenden Begleitmedikation mit topischen oder systemischen Kortikoiden. Sowohl systemische, als auch in geringerem Masse inhalative Kortikoide [15] können Gesamt-IgESpiegel reduzieren und somit theoretisch den saisonalen IgE„Boost“ abmildern. Somit reflektieren die von uns beobachteten Schwankungen des IgE-Spiegels einen weitgehend unverfälschten, maximalen „Boost“. Interessanterweise blieb der relative Anteil des spezifischen IgE am Gesamt-IgE während der Saison unverändert. Dies weist daraufhin, dass eine saisonale Exposition über verstärkte Produktion von T-Helfer (2)-Zytokinen [16] auch einen unselektiven, polyvalenten IgE-Anstieg triggert. Ein saisonaler Anstieg TH(2)-assoziierter Antikörper bei Patienten mit Autoimmunthyreoiditis während der Zedernpollensaison in Japan wurde kürzlich beschrieben [17]. Jedoch zeigten andere Autoren, dass eine saisonale Exposition bei Pollenallergikern nicht notwendigerweise mit einem Anstieg von spezifischem IgE gegen andere, z.B. perenniale Allergene einhergehen muss [18].

Wenngleich wir einen signifikanten saisonalen Anstieg des Gesamt- und spezifischen IgE bei allen Patienten nachweisen konnten, hätte dies in der Konsequenz bei der Mehrzahl der Patienten zu keiner Änderung der Omalizumab-Dosis führen müssen. Lediglich zwei Patienten fielen aus dem Dosisschema gänzlich heraus, bei den übrigen drei Patienten mit einer Änderung 


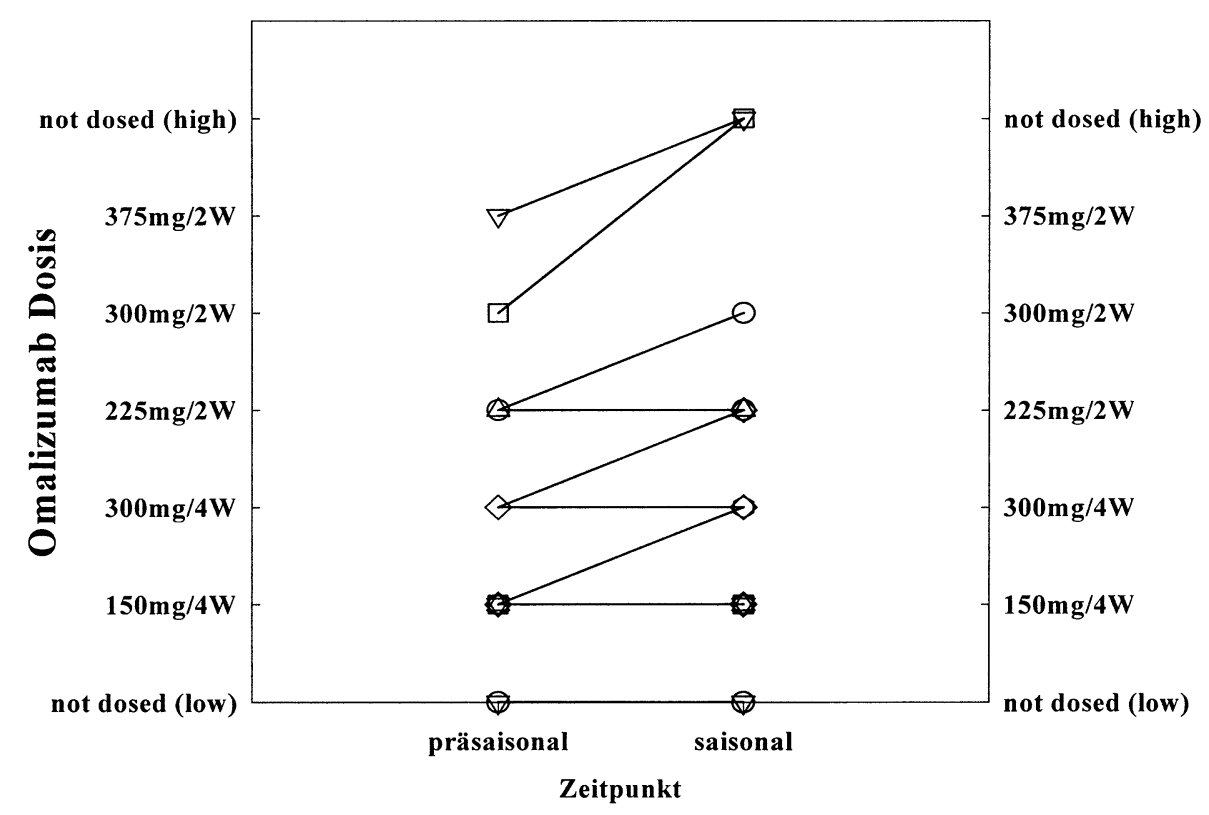

der Dosisstufe lagen die IgE-Werte jeweils 10,30 und $60 \mathrm{kU} / \mathrm{l}$ über der vorangehenden Stufe. Zumindest bei den hier untersuchten Patienten mit saisonaler Allergie erscheint es angesichts eines Anteils des spezifischen IgE am Gesamt-IgE von lediglich $\sim 12 \%$ daher unwahrscheinlich, dass die beobachteten Schwankungen auch unter Beibehaltung der Dosis zu einer relevanten Änderung der Therapiezuverlässigkeit geführt hätten. Dies belegt zum einen zwar die Praktikabilität des Dosierungsschemas von Omalizumab für die Mehrzahl der Patienten. Zum anderen wird jedoch anhand der geschilderten zwei Patienten deutlich, dass IgE-Spiegel in Einzelfällen erheblichen Schwankungen unterliegen können und daher die Gesamt-IgE-basierte Dosierung von Omalizumab mit einer gewissen Unsicherheit einhergehen kann. Es ist dabei theoretisch sowohl eine Über-, als auch eine Unterdosierung von Omalizumab denkbar. Wenngleich der hier zugrundeliegende Beipackzettel für Omalizumab aus Australien konstatiert, dass „.... there is no significant temporal variation in serum total IgE-levels", hat auch die Food and Drug Administration (FDA) bei der Protokollierung der Zulassung von Omalizumab in den USA angemerkt, dass bezüglich der Langzeitstabilität von Gesamt-IgE-Spiegeln noch offene Fragen existieren und die Herstellerfirma zu entsprechenden prospektiven Studien aufgefordert (http://www.fda.gov/cder/foi/ appletter/2003/omalgen062003L.htm).

Da für den Therapieerfolg die Höhe des freien IgE entscheidend sein dürfte, ist ein therapeutisches Monitoring von freiem IgE prinzipiell wünschenswert und notwendig. Kommerzielle Assays erfassen sowohl freies als auch komplexiertes IgE und sind daher zum Therapiemonitoring nicht geeignet. Diese Problematik wird zudem dadurch kompliziert, dass aufgrund der längeren Halbwertszeit der IgE/Omalizumab-Komplexe die Gesamt-IgESpiegel unter Therapie in herkömmlichen Assays sogar z.T. deutlich ansteigen, somit also diagnostisch unbrauchbar werden. Assays zur Messung von freiem IgE befinden sich derzeit in Entwicklung (Novartis, persönliche Kommunikation). Es steht zu hoffen, dass in Zukunft unter Monitoring von freiem IgE eine optimale Dosisanpassung in der Praxis erfolgen kann. Dies ist ins-
Abb. 3 Hypothetische Dosierung von Omalizumab bei saisonalen Allergikern auf Grundlage präsaisonaler oder saisonaler Gesamt-IgE-Spiegel. besondere auch vor dem Hintergrund der hohen Kosten einer Omalizumab-Behandlung von großer pharmakoökonomischer Bedeutung. Einige In-vitro-Untersuchungen weisen zudem daraufhin, dass es unter Omalizumab-Therapie zu einer Abregulation von hochaffinen IgE-Rezeptoren auf Basophilen kommt [19]. Dies wiederum könnte in der Konsequenz zu einer geringeren basalen IgE-Produktion unter Therapie führen und somit eine weitere Dosisreduktion ermöglichen. In der Praxis sollte vor Initiierung einer Behandlung mit Omalizumab im Einzelfall (ggf. auch mit wiederholten Gesamt-IgE-Bestimmungen) geprüft werden, ob evtl. vorhandene Schwankungen des IgE-Spiegels die therapeutische Dosis von Omalizumab beeinflussen würden. Im Zweifelsfalle sollte der jeweils höchste gemessene Wert als Grundlage für die Dosisberechnung herangezogen werden.

\section{Literatur}

${ }^{1}$ Barnes PJ. Anti-IgE therapy in asthma: rationale and therapeutic potential. Int Arch Allergy Immunol 2000; 123: 196-204

2 Buhl R, Soler M, Matz J et al. Omalizumab provides long-term control in patients with moderate-to-severe allergic asthma. Eur Respir J 2002; 20: $73-78$

${ }^{3}$ Busse WW, Corren J, Lanier BQ et al. Omalizumab, anti-IgE recombinant humanized monoclonal antibody, for the treatment of severe allergic asthma. J Allergy Clin Immunol 2001; 108: 184-190

${ }^{4}$ Milgrom H, Fick RB, Su JQ et al. Treatment of allergic asthma with monoclonal anti-IgE antibody. N Engl J Med 1999; 341: 1966-1973

${ }^{5}$ Adelroth E, Rak S, Haahtela T et al. Recombinant humanized mAb-E25, an anti-IgE $\mathrm{mAb}$, in birch pollen-induced seasonal allergic rhinitis. J Allergy Clin Immunol 2000; 106: 253-259

${ }^{6}$ Kuehr J, Brauburger J, Zielen S et al. Efficacy of combination treatment with anti-IgE plus specific immunotherapy in polysensitized children and adolescents with seasonal allergic rhinitis. J Allergy Clin Immunol 2002; 109: $274-280$

${ }^{7}$ Casale TB, Condemi JJ, LaForce C et al. Effect of omalizumab on symptoms of seasonal allergic rhinitis. JAMA 2001; 286: 2956-2967

${ }^{8}$ Casale TB, Bernstein IL, Busse WW et al. Use of an anti-Ige humanized monoclonal antobody in ragweed-induced allergic rhinits. J Allergy Clin Immunol 1997; 100: 110-121

${ }^{9}$ Malveaux FJ, Conroy MC, Adkinson Jr NF et al. IgE receptors on human basophils. Relationship to serum IgE concentration. J Clin Invest 1978; 62: 176-181 
${ }^{10}$ Xolair - package information leaflet. Australia, 2003

${ }^{11}$ Masamoto T, Ohashi Y, Nakai Y. Specific immunoglobulin E, interleukin-4, and soluble vascular cell adhesion molecule-1 in sera in patients with seasonal allergic rhinitis. Ann Otol Rhinol Laryngol 2004; 108: 169 - 176

12 Rossi RE, Monasterolo G, Operti D et al. Evaluation of IgE antibodies to recombinant pollen allergens ( $\mathrm{Phl} \mathrm{p} \mathrm{1,} \mathrm{Phl} \mathrm{p} \mathrm{2,} \mathrm{and} \mathrm{Phl} \mathrm{p} \mathrm{5)} \mathrm{in} \mathrm{a} \mathrm{random}$ sample of patients with specific IgE to Phleum pratense. Allergy 2004; 55: 181 - 184

13 Bland JM, Altman DC. Transformations, means, and confidence intervals. Br Med J 1996; 312: 1079

14 Boulet LP, Turcotte $\mathrm{H}$, Laprise $\mathrm{C}$ et al. Comparative degree and type of sensitization to common indoor and outdoor allergens in subjects with allergic rhinitis and/or asthma. Clin Exp Allergy 1997; 27: 52 - 59
${ }^{15}$ Corne J. Do inhaled corticosteroids reduce serum IgE levels? The answer is maybe but how relevant is the question? Clin Exp Allergy 1999; 29: $294-297$

16 Wosinska-Becler K, Plewako H, Hakansson L et al. Cytokine production in peripheral blood cells during and out of the pollen season in birch-allergic patients and non-allergic controls. Clin Exp Allergy 2004; 34: $123-130$

17 Takeoka K, Hidaka Y, Hanada $\mathrm{H}$ et al. Increase in serum levels of autoantibodies after attack of seasonal allergic rhinitis in patients with Graves' disease. Int Arch Allergy Immunol 2003; 132: 268 - 276

18 Ohashi Y, Nakai Y, Tanaka A et al. Seasonal rise in interleukin-4 during pollen season is related to seasonal rise in specific IgE for pollens but not for mites. Acta Otolaryngol 1998; 118: 243-247

19 MacGlashan DW, Bochner BS, Adelman DC et al. Down-regulation of FCeRI expression on human basophils during in vivo treatment of atopic patients with anti-IgE antibody. J Immunol 1997; 158: 1438-1445 